hep-th/0107145

SACLAY-SPHT-T01/68

\title{
U-duality from Matrix Membrane Partition Function
}

\author{
Fumihiko Sugino and Pierre Vanhove \\ Service de Physique Théorique, CEA-Saclay, \\ F-91191 Gif-sur-Yvette Cedex, France. \\ sugino, vanhove@spht.saclay.cea.fr
}

\begin{abstract}
We analyse supermembrane instantons (fully wrapped supermembranes) by computing the partition function of the three-dimensional supersymmetrical $\mathrm{U}(\mathrm{N})$ matrix model under periodic boundary conditions. By mapping the model to a cohomological field theory and considering a mass-deformation of the model, we show that the partition function exactly leads to the U-duality invariant measure factor entering supermembrane instanton sums. On the other hand, a computation based on the quasi-classical assumption gives the non U-duality invariant result of the zero-mode analysis by Pioline et al. [1]. This is suggestive of the importance of supermembrane self-interactions and shows a crucial difference from the matrix string case.
\end{abstract}

July 2001 


\section{Introduction}

The supermembrane (M2-brane) is a mysterious quantum object. The poor understanding of its three-dimensional world-volume theory makes difficult to consider it as a fundamental object. The classical relation between the Cremmer-Julia-Scherk supergravity [2] and the background fields for the supermembrane world-volume theory [3] is suggestive of the relation between a fundamental object and its low-energy effective action. In this case the only dimensionful parameter is the eleven-dimensional Planck length $\ell_{P}$. On the other hand, compactified supermembranes give rise to D-branes 四 therefore to non-perturbative string theory effects. In dimensions lower than eleven a new parameter (the eleventh radius $R_{11}$ interpreted as the string coupling constant [5]) allows one to make the difference between fundamental quantum excitations (the fundamental strings) and solitonic configurations (the D-branes).

In this setup semi-classical rules for classifying D-brane configurations can be derived [6,7]. The configurations are characterized by U-duality invariant number-theoretic functions $[8]$, associated with the bulk contribution of the Witten index for the effective $\mathrm{U}(\mathrm{N})$ supersymmetric matrix model description [9, 10, 11, 12]. These matrix models for the collective dynamics of $N$ D-branes are obtained by compactifying the $\mathrm{U}(\mathrm{N})$ supersymmetric quantum mechanics [13], originating from a $\mathrm{SU}(\mathrm{N})$ regularization of the light-cone Hamiltonian [14] for the supermembrane

$$
\mathcal{H}=\frac{1}{P_{0}^{+}} \int d^{2} \sigma \sqrt{w}\left[\frac{P^{a} P_{a}}{2 w}+\frac{1}{4}\left\{X^{a}, X^{b}\right\}^{2}-P_{0}^{+} \bar{\theta} \gamma_{-} \gamma_{a}\left\{X^{a}, \theta\right\}\right] .
$$

The purpose of this paper is to exhibit the origin of the U-duality group in M-theory compactified on a three-torus

$$
G_{U}=S l(3, \mathbb{Z}) \times S l(2, \mathbb{Z}),
$$

by considering Euclidean supermembranes wrapped over the three-torus. The $S l(3, \mathbb{Z})$ group corresponds to the area preserving group of isometries of the three-torus and $\operatorname{Sl}(2, \mathbb{Z})$ acts on the complex parameter $\Omega=C_{123}+i \mathrm{Vol}_{3}$ made from the v.e.v. of the three-form potential and the volume of the three-torus the supermembrane is fully wrapped on. The origin of the two groups in (1.2) is understood from the matrix model setup [15], as the geometrical $S l(3, \mathbb{Z})$ symmetry group of reparametrizations of the three-torus, and a quantum $S l(2, \mathbb{Z})$ symmetry group exchanging the different saddle-point contributions of the path integral. 
Counting multiply-wrapped Euclidean D-strings [7] consists of including all the supersymmetric maps of the D-string world-sheet onto the space-time compactification torus, modulo local and global reparametrizations. This rule corresponds to the classical saddlepoint of the path integral of the free sigma model for the D-string in the background of the Euclidean two-torus. A result re-obtained in [16] by computing the partition function (with the zero-modes subtracted) of the two-dimensional $\mathrm{U}(\mathrm{N})$ matrix string model. The two-dimensional matrix model can be written as a cohomological field theory for which the quasi-classical approximation is exact [17], and the problem reduces to sum over free singly connected long strings wrapping the two-torus [16].

We explain, in section 3 , that the three-dimensional matrix model does not have exact quasi-classics, therefore the free theory reduction is not enough. Summing only over the classical configurations of the three-dimensional matrix model seems to be equivalent to the zero-mode approach to the path integral over supermembrane configurations of Pioline et al.[1], where all the interactions in (1.1) were discarded. This approximation leads to the geometrical measure factor

$$
\hat{\mu}(N)=\sum_{n \mid N} n \sum_{p \mid(N / n)} p^{2},
$$

which counts the ways to map a volume- $N$ three-torus onto an Euclidean unit-volume target three-torus, modulo local reparametrizations. Unlike for the D-string case [7], this function (1.3) is not invariant under the full U-duality group (1.2) but is invariant under $S l(3, \mathbb{Z})$ alone. The correct counting of configurations of wrapped supermembranes is given by the number-theoretic function [1, 18]

$$
\mu(N)=\sum_{n \mid N} n .
$$

For $N$ a large prime number, $\hat{\mu}(N) \simeq N \mu(N)$ meaning that U-duality equivalent configurations were over-counted by the factor $N$ and $\mu(N)$ predicts the number of ground states for the supermembrane. The departure of $\hat{\mu}(N)$ from $N \times \mu(N)$ for finite values of $N$ shows that the problem is slightly more subtle than an over-counting.

We explain in section 4 that the difference between these two functions could be traced back to the presence of the interaction terms in the Hamiltonian (1.1). By considering massive deformations of the $\mathcal{N}_{3}=8$ supersymmetric three-dimensional $\mathrm{U}(\mathrm{N})$ matrix model 
into a $\mathcal{N}_{3}=2$ supersymmetric gauge theory in the cohomological field theory approach, the correct counting of configurations (1.4) will be derived.

We conclude, in section 5, with comments about a possible path integral approach to the supermembrane effects.

\section{The Matrix Model Description}

In order to obtain a matrix model description, we start with the system of $N$ Dparticles in (Euclidean) type IIA theory compactified on a two-torus $\mathcal{T}^{2}$ parametrized by $x^{9}$ and $x^{10}$. The D-particles are wrapped on the time direction $x^{10}$. Then, we consider the following duality sequences: $T_{9} S T_{9}$, where $S$ and $T_{9}$ stand, respectively, for the S-duality and the T-duality operation with respect to the direction $x^{9}$. As a result, we have the system of $N$ fundamental strings in (Euclidean) type IIA theory on $\mathcal{T}^{2}$, where the fundamental strings are fully wrapped on the $\mathcal{T}^{2}$. This is the same argument as in the derivation of matrix string theory [19] except that the direction $x^{10}$ is compactified. One then considers the matrix string theory compactified on a further $S^{1}$. By application of the argument for the compactification by Taylor [13], we obtain the maximally supersymmetric Yang-Mills theory with the gauge group $\mathrm{U}(\mathrm{N})$ in Euclidean three dimensions. It is composed by three gauge connections $A_{a}(a=1,2,3)$, seven adjoint scalars $X^{I}(I=1, \cdots, 7)$ and sixteen real adjoint fermions $\Psi_{\alpha}(a=1, \cdots, 16)$ :

$$
\begin{aligned}
S_{3 \mathrm{D}}[A, X, \Psi]=\frac{1}{g_{3 \mathrm{D}}^{2}} \int_{\mathcal{T}^{3}} d^{3} \sigma \operatorname{Tr}\left[\frac{1}{4} F_{a b} F^{a b}+\right. & \frac{1}{2}\left(D_{a} X^{I}\right)^{2}+\frac{i}{2} \Psi^{T} \Gamma^{a} D_{a} \Psi \\
& \left.-\frac{1}{4}\left[X^{I}, X^{J}\right]^{2}+\frac{1}{2} \Psi^{T} \Gamma^{I}\left[X_{I}, \Psi\right]\right] .
\end{aligned}
$$

As usual $D_{a}=\partial_{a}-i A_{a}$. The coupling constant $g_{3 \mathrm{D}}^{2}$ has the dimension (length) ${ }^{-1}$. This model has sixteen real supercharges $\left(\mathcal{N}_{3}=8\right)$ and is invariant under the $\operatorname{Sl}(3, \mathbb{Z})$ group of reparametrizations of the Euclidean rectangular three-torus $\mathcal{T}^{3}$ with lengths $\left(R_{1}, R_{2}, R_{3}\right)$. This group corresponds to the $S l(3, \mathbb{Z})$ appearing in the U-duality group (1.2). The volume of this torus will be denoted by $\mathcal{V}_{o}=R_{1} R_{2} R_{3}$.

As in the matrix string case [16], the amplitude of supermembranes fully wrapped on the three-torus in M-theory corresponds to the partition function of the super Yang-Mills theory:

$$
Z_{\mathcal{N}_{3}=8}^{\mathrm{U}(\mathrm{N})}\left[\mathcal{V}_{o}\right]=\int \frac{[\mathcal{D} A]}{\operatorname{Vol}(U(N))}[\mathcal{D} X][\mathcal{D} \Psi] \delta\left(X^{(0)}\right) \delta\left(\Psi^{(0)}\right) e^{-S_{3 \mathrm{D}}[A, X, \Psi]}
$$


where all the fields obey periodic boundary conditions and the zero-mode subtractions are defined as

$$
\delta\left(X^{(0)}\right) \equiv \prod_{I=1}^{7} \delta\left(\frac{\operatorname{Tr} \int_{\mathcal{T}^{3}} d^{3} \sigma X_{I}}{\sqrt{N \mathcal{V}_{o}}}\right), \quad \delta\left(\Psi^{(0)}\right) \equiv \prod_{\alpha=1}^{16} \delta\left(\frac{\operatorname{Tr} \int_{\mathcal{T}^{3}} d^{3} \sigma \Psi_{\alpha}}{\sqrt{N \mathcal{V}_{o}}}\right)
$$

The path integral measures are normalized using the natural metric on the space of small deformations $\delta \varphi$, for $\varphi$ meaning the gauge connection $A_{a}$ or the matter field $X^{I}$ and $\Psi_{\alpha}$ :

$$
\int[\mathcal{D} \delta \varphi] \exp \left(-\frac{1}{2 g_{3 \mathrm{D}}^{2}} \int_{\mathcal{T}^{3}} d^{3} \sigma \operatorname{Tr}(\delta \varphi)^{2}\right)=1
$$

\section{The Quasi-Classical Calculation}

We show that, if we assume that only the variables along the flat directions of the potential are relevant and contribute to the partition function (as for the quasi-classical assumption considered in [16]), we obtain the non U-duality invariant measure $\hat{\mu}(N)$ given in (1.3). The analysis is performed using the method of [16] with all the modifications needed for the three-dimensional case.

We project on the flat directions 1

$$
\begin{aligned}
& \operatorname{Tr}\left(\left[A_{a}, A_{b}\right]^{2}\right)=0, \operatorname{Tr}\left(\left[A_{a}, X^{I}\right]^{2}\right)=0, \quad \operatorname{Tr}\left(\left[X^{I}, X^{J}\right]^{2}\right)=0, \\
& \operatorname{Tr}\left(\Psi^{T} \Gamma^{I}\left[X_{I}, \Psi\right]\right)=0, \quad \operatorname{Tr}\left(\Psi^{T} \Gamma^{a}\left[A_{a}, \Psi\right]\right)=0 .
\end{aligned}
$$

That results into breaking the $\mathrm{U}(\mathrm{N})$ gauge symmetry to $\mathrm{U}(1)^{N}$. The fields $\Phi=\left\{D_{a}=\right.$ $\left.\partial_{a}-i A_{a}, X^{I}, \Psi^{\alpha}\right\}$ can be simultaneously diagonalized by a unitary matrix $V\left(\sigma^{1}, \sigma^{2}, \sigma^{3}\right)$ such that

$$
\Phi\left(\sigma^{1}, \sigma^{2}, \sigma^{3}\right)=V^{-1}\left(\sigma^{1}, \sigma^{2}, \sigma^{3}\right) \Phi^{D}\left(\sigma^{1}, \sigma^{2}, \sigma^{3}\right) V\left(\sigma^{1}, \sigma^{2}, \sigma^{3}\right)
$$

with $\Phi^{D}=\operatorname{diag}\left\{\Phi_{1}, \cdots, \Phi_{N}\right\}$, giving rise to the twisted boundary conditions 116

$$
\begin{array}{ll}
\Phi^{D}\left(R_{1}, \sigma^{2}, \sigma^{3}\right)=S^{-1} \Phi^{D}\left(0, \sigma^{2}, \sigma^{3}\right) S, & S=V\left(0, \sigma^{2}, \sigma^{3}\right) V^{-1}\left(R_{1}, \sigma^{2}, \sigma^{3}\right) \\
\Phi^{D}\left(\sigma^{1}, R_{2}, \sigma^{3}\right)=T^{-1} \Phi^{D}\left(\sigma^{1}, 0, \sigma^{3}\right) T, & T=V\left(\sigma^{1}, 0, \sigma^{3}\right) V^{-1}\left(\sigma^{1}, R_{2}, \sigma^{3}\right) \\
\Phi^{D}\left(\sigma^{1}, \sigma^{2}, R_{3}\right)=U^{-1} \Phi^{D}\left(\sigma^{1}, \sigma^{2}, 0\right) U, & U=V\left(\sigma^{1}, \sigma^{2}, 0\right) V^{-1}\left(\sigma^{1}, \sigma^{2}, R_{3}\right)
\end{array}
$$

where the matrices $S, T$ and $U$ act as permutation operators on the eigenvalues of the fields $\Phi$. For consistency of the boundary conditions, the matrices must be mutually

1 This limit corresponds to rescaling the fields by a factor of $g_{3 \mathrm{D}}$ and sending $g_{3 \mathrm{D}} \rightarrow \infty$. 
commuting. Each triplet of permutations $(S, T, U)$ describes coverings of the three-torus $\mathcal{T}^{3}$ with, in general, several disconnected components. Each component is interpreted as a fully wrapped supermembrane over the three-torus. Because each component has sixteen fermionic zero-modes, saturation of the fermionic zero-modes in the partition function selects singly connected configurations [16]. They correspond to various states of a single long supermembrane wrapping $N$ times the three-torus, reducing the model to a free $\mathrm{U}(1)$ matrix model on a three-torus of extended size $N \mathcal{V}_{o}$ with still sixteen real supercharges $\left(\mathcal{N}_{3}=8\right)$. The large torus is characterized by the matrix $M=\left[m_{i j}\right]_{1 \leq i, j \leq 3}$ with the all entries being integers and $\operatorname{det} M=N$, i.e. it is spaned by the three vectors $\vec{\omega}_{a}=$ $\left(m_{a 1} R_{1}, m_{a 2} R_{2}, m_{a 3} R_{3}\right)$. The periodicity of the large torus leads to

$$
S^{m_{a 1}} T^{m_{a 2}} U^{m_{a 3}}=1 \quad \forall a \in\{1,2,3\} .
$$

Here, all these equations are not independent. Using $S l(3, \mathbb{Z})$ transformations, they can be reduced to

$$
U^{p}=T^{n} U^{k}=S^{m} T^{j} U^{l}=1,
$$

with $N=m n p, j=0, \cdots, n-1$ and $k, l=0, \cdots, p-1$. Correspondingly, the matrix $M$ becomes

$$
M=\left(\begin{array}{ccc}
m & j & l \\
0 & n & k \\
0 & 0 & p
\end{array}\right),
$$

which is a representative of classes modulo the left-action of $S l(3, \mathbb{Z})$. Also, the three vectors spanning the corresponding torus $\widetilde{\mathcal{T}}^{3}$ are

$$
\vec{\omega}_{1}=\left(m R_{1}, j R_{2}, l R_{3}\right), \quad \vec{\omega}_{2}=\left(0, n R_{2}, k R_{3}\right), \quad \vec{\omega}_{3}=\left(0,0, p R_{3}\right)
$$

An explicit solution to equations (3.5) is

$$
\begin{aligned}
& U=P_{N}^{m n}, \quad T=P_{N}^{-m k}\left(\begin{array}{ccc}
P_{x}^{m} & & \\
& \ddots & \\
& & P_{x}^{m}
\end{array}\right), \\
& S=P_{N}^{k j-n l}\left(\begin{array}{ccc}
P_{x}^{-j} & & \\
& \ddots & \\
& & P_{x}^{-j}
\end{array}\right)\left(\begin{array}{ccc}
P_{y} & & \\
& \ddots & \\
& & P_{y}
\end{array}\right),
\end{aligned}
$$


where $x=\operatorname{gcd}(m n, m k, k j-n l), y=\operatorname{gcd}(m, j, k j-n l)$ and for any integer $i$ we define $\operatorname{gcd}(i, 0)=i . \quad P_{u}$ represents a $u \times u$ matrix of a cyclic permutation:

$$
P_{u}=\left(\begin{array}{ccccc}
0 & 1 & 0 & \cdots & 0 \\
0 & 0 & 1 & \cdots & 0 \\
\vdots & \vdots & \vdots & & \vdots \\
0 & 0 & 0 & \cdots & 1 \\
1 & 0 & 0 & \cdots & 0
\end{array}\right)
$$

$P_{N}^{m n}, P_{N}^{-m k}$ and $P_{N}^{k j-n l}$ define a covering with $x$ disconnected components, but the mutually commuting $S, T$ and $U$ represent a single component covering. $⿴$. The above solution is a representative modulo appropriate permutations acting on the basis of $S, T, U$. The number of these degrees of freedom is counted to be $(N-1)$ ! as in the matrix string case [16].

In the quasi-classical limit, the partition function reduces to the sum over the partition functions of $U(1)$ supersymmetric gauge theory with the zero-modes subtracted defined on the various tori (3.7):

$$
\begin{aligned}
Z_{\mathcal{N}_{3}=8}^{\mathrm{U}(\mathrm{N})}\left[\mathcal{V}_{o}\right] & =\frac{(N-1) !}{N !} \sum_{\substack{M \\
\operatorname{det} M=N}} Z_{[M]}^{\mathrm{U}(1)}\left[N \mathcal{V}_{o}\right], \\
Z_{[M]}^{\mathrm{U}(1)}\left[N \mathcal{V}_{o}\right] & =\int \frac{[\mathcal{D} A]}{\operatorname{Vol}(U(1))}[\mathcal{D} X][\mathcal{D} \Psi] \delta\left(X^{(0)}\right) \delta\left(\Psi^{(0)}\right) e^{-S^{\mathrm{U}(1)}},
\end{aligned}
$$

where

$$
S^{\mathrm{U}(1)}=\frac{1}{g_{3 D}^{2}} \int_{\widetilde{\mathcal{T}}^{3}} d^{3} \tilde{\sigma} \sqrt{g}\left[\frac{1}{4} g^{a b} g^{c d} F_{a c} F_{b d}+\frac{1}{2} g^{a b} \partial_{a} X_{I} \partial_{b} X_{I}+\frac{i}{2} \Psi^{T} \Gamma^{a} \partial_{a} \Psi\right] .
$$

We introduced the coordinates $\tilde{\sigma}^{a}$ ranging from 0 to $\left|\vec{\omega}_{a}\right|$ with the constant metric $g_{a b}$ such that $\int_{\widetilde{\mathcal{T}}^{3}} d^{3} \tilde{\sigma} \sqrt{g}=N \mathcal{V}_{o}$. The denominator in the first formula $N$ ! comes from the volume of the permutation group $S_{N}$ which is a part of the original gauge group $\mathrm{U}(\mathrm{N})$. All the fields

2 In the matrix string case, the solution (26) in ref. [16] should be corrected so that it represents a single component covering. For the equations $T^{n}=T^{j} S^{m}=1$ with $N=m n$ and $j=0, \cdots, n-1$, a correct solution such that $S$ and $T$ are mutually commuting and form a singly connected covering is as follows:

$$
T=P_{N}^{m}, \quad S=P_{N}^{-j}\left(\begin{array}{ccc}
P_{v} & & \\
& \ddots & \\
& & P_{v}
\end{array}\right)
$$

where $v=\operatorname{gcd}(m, j)$. 
except nontrivial flux sectors for the gauge field enjoy the periodic boundary condition on the torus (3.7). The contributions from the gauge and the matter fields factorize as

$$
Z_{[M]}^{\mathrm{U}(1)}\left[N \mathcal{V}_{o}\right]=Z_{[M]}^{\mathrm{U}(1)-\text { gauge }}\left[N \mathcal{V}_{o}\right] \times\left(2 \pi g_{3 \mathrm{D}}^{2} \operatorname{det}^{\prime} \square\right)^{\frac{8}{2}-\frac{7}{2}}
$$

where the last factor comes from the integration over the sixteen fermions and the seven scalars. The prime means the omission of zero-modes of the Laplacian $\square \equiv-g^{a b} \partial_{a} \partial_{b}$. The first factor is the partition function for the $\mathrm{U}(1)$ gauge theory part, which we now evaluate.

At first, we consider the flux sectors for the gauge field. In order to do so, it is convenient to return to the description of the $\mathrm{U}(1)^{N}$-theory on the original torus. The fluxes on the original torus are quantized by the first Chern numbers as

$$
\int d \sigma^{a} d \sigma^{b} \operatorname{Tr} F_{a b}^{D}=2 \pi n_{a b}, \quad n_{a b}=-n_{b a} \in \mathbb{Z},
$$

where the superscript $D$ means a diagonal matrix. Thus, we can rewrite the $\mathrm{U}(1)^{N}$ gauge field as $A_{a}^{D}=-\sum_{b(<a)} f_{a b} \sigma^{b} \mathbf{1}_{N}+\tilde{A}_{a}^{D}$, where $f_{a b}=2 \pi \frac{n_{a b}}{N R_{a} R_{b}}$ represents a constant magnetic flux and $\tilde{A}_{a}^{D}$ does not generate fluxes globally. The value of the classical action for the flux is easily evaluated as

$$
S^{\text {flux }}=\frac{(2 \pi)^{2}}{2 N g_{3 \mathrm{D}}^{2} \mathcal{V}_{o}}\left[q_{1}^{2} R_{1}^{2}+q_{2}^{2} R_{2}^{2}+q_{3}^{2} R_{3}^{2}\right]
$$

where $q_{a}$ is an integer dual to $n_{a b}$. $\tilde{A}_{a}^{D}$ corresponds to a $\mathrm{U}(1)$ gauge field on the extended torus obeying the periodic boundary condition.

Next we consider orthogonal decompositions of the $\mathrm{U}(1)^{N}$ gauge field with respect to the inner product on the space of connections defined by

$$
\left(\delta A_{(1)}^{D}, \delta A_{(2)}^{D}\right) \equiv \int_{\mathcal{T}^{3}} d^{3} \sigma \delta^{a b} \operatorname{Tr}\left(\delta A_{(1) a}^{D} \delta A_{(2) b}^{D}\right)
$$

Note that the flux sector is discrete and it does not contribute to the continuous variation $\delta A^{D}$. It is easy to see that the following decomposition is possible: $\delta A_{a}^{D}=\delta \tilde{A}_{a}^{D}=$ $\delta \bar{A}_{a}^{D}+\delta \hat{A}_{a}^{D}+\partial_{a} \delta \phi^{D}$ where $\hat{A}_{a}^{D}$ is a quantum fluctuation satisfying the Lorentz gauge condition $\partial \cdot \hat{A}^{D}=0$ and $\int_{\mathcal{T}^{3}} d^{3} \sigma \operatorname{Tr} \hat{A}_{a}^{D}=0$ and $\phi^{D}$ is a $\mathrm{U}(1)^{N}$ gauge function connected to the identity, with $\int_{\mathcal{T}^{3}} d^{3} \sigma \operatorname{Tr} \phi^{D}=0.3 \bar{A}_{a}^{D}$ is a constant zero-mode (a flat connection),

3 Since the constant zero-modes of $\phi^{D}$ cause no gauge transformation, we do not consider. 
which is immediately seen to be proportional to the unit matrix: $\bar{A}_{a}^{D}=c_{a} \mathbf{1}_{N}$ due to the periodic boundary conditions modulo $S_{N}$ permutations. The measure becomes

$$
\frac{\left[\mathcal{D} \tilde{A}^{D}\right]}{\operatorname{Vol}\left(U(1)^{N}\right)}=\left(\prod_{a=1}^{3} d c_{a}\right) \frac{\left[\mathcal{D} \hat{A}^{D}\right]\left[\mathcal{D} \partial \phi^{D}\right]}{\operatorname{Vol}\left(U(1)^{N}\right)}
$$

Because the gauge zero-mode $c_{a}$ lives on a circle of circumference $\frac{2 \pi}{R_{a}}$, the integrals over the zero-modes give the result

$$
\int \prod_{a=1}^{3} d c_{a}=\frac{(2 \pi)^{3}}{\mathcal{V}_{o}}
$$

Returning to the description of the U(1)-theory on the extended torus, we have

$$
\begin{aligned}
& Z_{[M]}^{\mathrm{U}(1)-\text { gauge }}\left[N \mathcal{V}_{o}\right]=\left(\sum_{\text {flux }} e^{-S^{\mathrm{flux}}}\right) \frac{(2 \pi)^{3}}{\mathcal{V}_{o}} \\
& \times \int[\mathcal{D} \hat{A}] \frac{[\mathcal{D} \phi]}{\operatorname{Vol}(U(1))}\left(\operatorname{det}^{\prime} \square\right)^{\frac{1}{2}} \exp \left(-\frac{1}{4 g_{3 \mathrm{D}}^{2}} \int_{\widetilde{\mathcal{T}}^{3}} d^{3} \tilde{\sigma}^{a} \hat{F}_{a b} \hat{F}^{a b}\right),
\end{aligned}
$$

where $\hat{F}_{a b}$ is a field strength corresponding to $\hat{A}_{a} . \hat{A}_{a}$ and $\phi$ are the variables of the $\mathrm{U}(1)$ gauge theory corresponding to $\hat{A}^{D}$ and $\phi^{D}$, respectively.

From the usual definition of the gauge volume $\operatorname{Vol}(U(1))$ which concerns elements connected to the identity and includes the constant modes of the gauge function, we have

$$
\int[\mathcal{D} \phi]=\frac{\operatorname{Vol}(U(1))}{2 \pi}
$$

We dualize the field strength into a vector $\hat{f}=\star \hat{F}=\star d \hat{A}$, and consider a change of the variables from $\hat{A}$ to $\hat{f}$. The Jacobian for this change is computed by remarking that the inner product

$$
(\delta \hat{f}, \delta \hat{f})=\int_{\widetilde{\mathcal{T}}^{3}} d^{3} \tilde{\sigma} \sqrt{g} g_{a b} \delta \hat{f}^{a} \delta \hat{f}^{b}=\int_{\widetilde{T}^{3}} d^{3} \tilde{\sigma} \sqrt{g} \delta \hat{A}^{a} \square P_{a}^{b} \delta \hat{A}_{b}
$$

implies $[\mathcal{D} \hat{f}]=\left[\operatorname{det}^{\prime} \square P_{a}^{b}\right]^{1 / 2}[\mathcal{D} \hat{A}]$ with $P_{a}{ }^{b}$ being the projection operator into transverse directions: $P_{a}{ }^{b}=\delta_{a}{ }^{b}+\frac{\partial_{a} \partial^{b}}{\square}$. Since $P_{a}{ }^{b}$ has the eigenvalues 0 and 1 with multiplicity 1 and 2 respectively, we have $[\mathcal{D} \hat{f}]=\left(\operatorname{det}^{\prime} \square\right)[\mathcal{D} \hat{A}]$. The last factor of the partition function $(3.19)$ becomes

$$
\frac{1}{2 \pi} \int[\mathcal{D} \hat{f}]\left(\operatorname{det}^{\prime} \square\right)^{\frac{1}{2}-1} \exp \left(-\frac{1}{2 g_{3 \mathrm{D}}^{2}} \int_{\tilde{\mathcal{T}}^{3}} d^{3} \tilde{\sigma} \sqrt{g} \hat{f}_{a} \hat{f}^{a}\right)
$$


Noticing that $\hat{f}$ has no zero-modes, from the normalization (2.4) follows

$$
\int[\mathcal{D} \hat{f}] \exp \left(-\frac{1}{2 g_{3 \mathrm{D}}^{2}} \int_{\widetilde{\mathcal{T}}^{3}} d^{3} \tilde{\sigma} \sqrt{g} \hat{f}_{a} \hat{f}^{a}\right)=\left(2 \pi g_{3 \mathrm{D}}^{2}\right)^{-3 / 2}
$$

Collecting everything, the determinant factors of the Laplacian are cancelled, the partition function (2.2) in the quasi-classical approximation becomes

$$
Z_{\mathcal{N}_{3}=8}^{\mathrm{U}(\mathrm{N})}\left[\mathcal{V}_{o}\right]=\frac{2 \pi}{N g_{3 \mathrm{D}}^{2} \mathcal{V}_{O}} \sum_{\substack{[M] \\ \operatorname{det} M=N}}\left(\sum_{\text {flux }} e^{-S^{\text {flux }}}\right)
$$

In the M-theory side, the fluxes are interpreted as Kaluza-Klein states (wrapped supergravitons) dissolved in the wrapped membrane. Since the flux sum is independent of the (discretized) "moduli" $M$ of $\widetilde{\mathcal{T}}^{3}$, the sum over the "moduli" leads to the number theoretic factor $(1.3)$ as

$$
\sum_{\substack{[M] \\ \operatorname{det} M=N}} 1=\sum_{m n p=N} n p^{2}=\sum_{n \mid N} n \sum_{p \mid(N / n)} p^{2}=\hat{\mu}(N)
$$

which counts the number of wrapping a size- $N \tilde{\mathcal{T}}^{3}$ over a unit-volume $\mathcal{T}^{3}$ up to local reparametrizations. It should be noticed that the origin of $\hat{\mu}(N)$ from the sum over the "moduli" is same as in the calculation in ref. [1].

Finally we obtain the result of the quasi-classical calculation as

$$
\begin{aligned}
\left.Z_{\mathcal{N}_{3}=8}^{\mathrm{U}(\mathrm{N})}\left[\mathcal{V}_{o}\right]\right|_{\text {quasi-classical }}= & \frac{2 \pi}{g_{3 \mathrm{D}}^{2} \mathcal{V}_{o}} \frac{\hat{\mu}(N)}{N} \\
& \times \sum_{q_{i} \in \mathbb{Z}} \exp \left(-\frac{(2 \pi)^{2}}{2 N g_{3 \mathrm{D}}^{2} \mathcal{V}_{o}}\left[q_{1}^{2} R_{1}^{2}+q_{2}^{2} R_{2}^{2}+q_{3}^{2} R_{3}^{2}\right]\right)
\end{aligned}
$$

We should remark that the result derived from the quasi-classical assumption respects the $S l(3, \mathbb{Z})$ symmetry of the model but breaks the $S l(2, \mathbb{Z})$ symmetry, as can be seen on the end result (3.25).

\section{The Cohomological Field Theory Approach}

In this section we follow the method of [17] (for different approaches to the cohomological matrix models see for instance [20,21]) that consists in deforming a three-dimensional cohomological field theory with sixteen real supercharges, which is equivalent to the matrix membrane model, by adding a mass perturbation such that the classical saddle point 
of the deformed theory is a three-dimensional gauge theory with four real supercharges $\left(\mathcal{N}_{3}=2\right)$. the results from the two methods coincide, because the theory after the mass deformation is an $\mathcal{N}_{2}=2$ super Yang-Mills theory, which can again be written as a cohomological field theory [17], and the calculation can be completely reduced to a classical saddle point problem. As we will see, the major difference between the matrix string and the matrix membrane models is that the mass-deformed matrix membrane model is not equivalent to a cohomological model any longer [17], therefore the computation will not entirely localize on the classical configurations.

The overall $\mathrm{U}(1)$ part of the gauge group $\mathrm{U}(\mathrm{N})$ decouples and can be treated as a free field theory. We use the mass deformation method on the remaining non-Abelian part of the partition function (2.2) as in [17]:

$$
Z_{\mathcal{N}_{3}=8}^{\mathrm{U}(\mathrm{N})}\left[\mathcal{V}_{o}\right]=Z_{\mathcal{N}_{3}=8}^{\mathrm{U}(1)}\left[\mathcal{V}_{o}\right] \times Z_{\mathcal{N}_{3}=8}^{\mathrm{SU}(\mathrm{N}) / \mathbb{Z}_{\mathrm{N}}}\left[\mathcal{V}_{o}\right]
$$

$Z^{\mathrm{U}(1)}$ denotes the partition function of the overall $\mathrm{U}(1)$ of the $\mathrm{U}(\mathrm{N})$ theory with the zeromode delta functions (2.3) inserted. The gauge field is expanded as $A_{a}=A_{a}^{\mathrm{U}(1)} \mathbf{1}_{N}+A_{a}^{r} T^{r}$, where $T^{r}$ 's span a basis of SU(N)-generators. The matter fields are decomposed similarly. First, we consider the partition function of the SU(N) theory $Z_{\mathcal{N}_{3}=8}^{\mathrm{SU}(\mathrm{N})}\left[\mathcal{V}_{o}\right]$ (in the zero 't Hooft discrete flux sector). The action is exactly in the form of the dimensional reduction of four-dimensional $\mathcal{N}_{4}=4 \mathrm{SU}(\mathrm{N})$ supersymmetric Yang-Mills theory, so the argument below is a dimensionally reduced version of the four-dimensional theory. We map the theory to a cohomological field theory by twisting [23] and calculate the partition function by adding the mass-perturbation

$$
\Delta S=-\left.\frac{m_{3 \mathrm{D}}}{2 \sqrt{2} g_{3 \mathrm{D}}^{2}} \int_{\mathcal{T}^{3}} d^{3} \sigma \operatorname{Tr}\left(\Phi_{1}^{2}+\Phi_{2}^{2}+\Phi_{3}^{2}\right)\right|_{\theta \theta}+\text { h.c. }
$$

where $\Phi_{s}(s=1,2,3)$ represents the chiral superfields in the four-dimensional $\mathcal{N}_{4}=1$ superfield formalism. After integrating out the chiral superfields in the mass-perturbed theory, contributions to the path integral localize on classical vacua described by threedimensional $\mathcal{N}_{3}=2$ supersymmetric $\mathrm{SU}(\mathrm{n}) \otimes \mathbb{Z}_{m}$ gauge theory with $N=m n$ [17]. As a result, the partition function $Z_{\mathcal{N}_{3}=8}^{\mathrm{SU}(\mathrm{N})}\left[\mathcal{V}_{o}\right]$ becomes

$$
Z_{\mathcal{N}_{3}=8}^{\mathrm{SU}(\mathrm{N})}\left[\mathcal{V}_{o}\right]=\sum_{m n=N} Z^{\mathrm{SU}(\mathrm{n}) \otimes \mathbb{Z}_{\mathrm{m}}}\left[\mathcal{V}_{o}\right]=\sum_{m n=N} m^{2} Z_{\mathcal{N}_{3}=2}^{\mathrm{SU}(\mathrm{n})}\left[\mathcal{V}_{o}\right]
$$

4 For a similar analysis in the context of super Yang-Mills quantum mechanics, see [22]. 
where the factor $m^{2}=m^{3-1}$ comes from the summation over the flat $\mathbb{Z}_{m}$-bundles.

The three-dimensional $\mathcal{N}_{3}=2 \mathrm{SU}(\mathrm{n})$ gauge theory with the periodic boundary conditions, has a single Higgs scalar field with a continuous spectrum beginning at zero-energy. Therefore, the relation between $Z_{\mathcal{N}_{3}=2}^{\mathrm{SU}(\mathrm{n})}\left[\mathcal{V}_{o}\right]$ and the Witten index is unclear. Seeing the three-dimensional gauge model as the dimensional reduction of the four-dimensional gauge theory, the partition function $Z_{\mathcal{N}_{3}=2}^{\mathrm{SU}(\mathrm{n})}\left[\mathcal{V}_{o}\right]$ is identified with the bulk part of the Witten index for the four-dimensional $\mathrm{SU}(\mathrm{n})$ gauge theory with four real supercharges $\left(\mathcal{N}_{4}=1\right)$ :

$$
I_{W}^{\mathcal{N}_{4}=1}(S U(n)) \equiv \lim _{\beta \rightarrow \infty} \operatorname{Str}\left(e^{-\beta H_{4 \mathrm{D}}}\right)=\lim _{\beta \rightarrow 0} \operatorname{Str}\left(e^{-\beta H_{4 \mathrm{D}}}\right)+\delta I_{W}
$$

where the fields obey the periodic boundary condition with respect to all the four directions. As will be seen just below, the modes which are constant with respect to the time variable dominate the supertrace term of the right hand side, in the limit $\beta \rightarrow 0$. It will result the three-dimensional model with the periodic boundary condition we are considering. However, the four-dimensional gauge theory in the finite box has a discrete spectrum, so the deficit term $\delta I_{W}$ vanishes. The first term in r.h.s. can be regarded as the partition function of the four-dimensional theory, where the fourth direction is a tiny circle of length $\beta: \lim _{\beta \rightarrow 0} Z_{\mathcal{N}_{4}=1}^{\mathrm{SU}(\mathrm{n})}\left[\mathcal{V}_{o} \times[0, \beta]\right]$. The action of the $\mathcal{N}_{4}=1$ four-dimensional $\mathrm{SU}(\mathrm{n})$ gauge model is

$$
S_{4 \mathrm{D}}=\int_{0}^{\beta} d t \int_{\mathcal{T}^{3}} d^{3} \sigma\left[\frac{1}{g_{4 \mathrm{D}}^{2}} \operatorname{Tr}\left(\frac{1}{4} F_{a b} F^{a b}+\frac{i}{2} \bar{\lambda} \Gamma \cdot \partial \lambda\right)\right]
$$

In the limit $\beta \rightarrow 0$ with $g_{3 \mathrm{D}}^{-2}=\beta g_{4 \mathrm{D}}^{-2}$ kept fixed, because of the discreteness of its spectrum the theory smoothly flows to the three-dimensional $\mathcal{N}_{3}=2$ theory. the value of the Witten index is known from ref. [24] to be $I_{W}^{\mathcal{N}_{4}=1}(S U(n))=n$. Therefore, we get

$$
Z_{\mathcal{N}_{3}=2}^{\mathrm{SU}(\mathrm{n})}\left[\mathcal{V}_{o}\right]=n
$$

Plugging this result into (4.3), we obtain for the $\mathrm{SU}(\mathrm{N}) / \mathbb{Z}_{N}$ partition function with periodic boundary conditions

$$
Z_{\mathcal{N}_{3}=8}^{\mathrm{SU}(\mathrm{N}) / \mathbb{Z}_{\mathrm{N}}}\left[\mathcal{V}_{o}\right]=\frac{1}{N^{2}} Z_{\mathcal{N}_{3}=8}^{\mathrm{SU}(\mathrm{N})}\left[\mathcal{V}_{o}\right]=\frac{1}{N^{2}} \sum_{m n=N} m^{2} n=\frac{1}{N} \sum_{m \mid N} m=\frac{1}{N} \mu(N)
$$

which is the U-duality invariant function (1.4).

5 This is a similar argument as in [9] for the super Yang-Mills quantum mechanics. However, our case is more obvious because the spectrum is discrete. 
Next, we consider the contribution of the overall $\mathrm{U}(1)$-part $Z^{\mathrm{U}(1)}\left[\mathcal{V}_{o}\right]$. The action is

$$
S^{\mathrm{U}(1)}=\frac{N}{g_{3 \mathrm{D}}^{2}} \int_{\mathcal{T}^{3}} d^{3} \sigma\left[\frac{1}{4}\left(F_{a b}^{\mathrm{U}(1)}\right)^{2}+\frac{1}{2}\left(\partial_{a} X^{\mathrm{U}(1)}\right)^{2}+\frac{i}{2} \Psi^{\mathrm{U}(1) T} \Gamma_{a} \partial_{a} \Psi^{\mathrm{U}(1)}\right] .
$$

The path integral is performed with the normalizations (2.4):

$$
\int\left[\mathcal{D} \delta \varphi^{\mathrm{U}(1)}\right] \exp \left(-\frac{N}{2 g_{3 D}^{2}} \int_{\mathcal{T}^{3}} d^{3} \sigma\left(\delta \varphi^{\mathrm{U}(1)}\right)^{2}\right)=1 .
$$

Contributions from the fluxes and the flat connections are evaluated by the same way as in the quasi-classical case (3.15) and (3.18). The integrals for the matter part give the factor $\left(2 \pi g_{3 \mathrm{D}}^{2} \operatorname{det}^{\prime} \square\right)^{1 / 2}$, and the same procedure as in the quasi-classical computation leads to the result

$$
Z^{\mathrm{U}(1)}\left[\mathcal{V}_{o}\right]=\frac{2 \pi}{g_{3 \mathrm{D}}^{2} \mathcal{V}_{o}} \sum_{q_{i} \in \mathbb{Z}} \exp \left(-\frac{(2 \pi)^{2}}{2 N g_{3 \mathrm{D}}^{2} \mathcal{V}_{o}}\left[q_{1}^{2} R_{1}^{2}+q_{2}^{2} R_{2}^{2}+q_{3}^{2} R_{3}^{2}\right]\right)
$$

where $q_{i}$ 's represent the fluxes.

Now we find the final form of the cohomological field theory calculation as

$$
Z_{\mathcal{N}_{3}=8}^{\mathrm{U}(\mathrm{N})}\left[\mathcal{V}_{o}\right]=\frac{2 \pi}{g_{3 \mathrm{D}}^{2} \mathcal{V}_{o}} \frac{\mu(N)}{N} \sum_{q_{i} \in \mathbb{Z}} \exp \left(-\frac{(2 \pi)^{2}}{2 N g_{3 \mathrm{D}}^{2} \mathcal{V}_{o}}\left[q_{1}^{2} R_{1}^{2}+q_{2}^{2} R_{2}^{2}+q_{3}^{2} R_{3}^{2}\right]\right)
$$

In the zero-flux sector, the result (4.11) reproduces the U-duality invariant counting (1.4) of wrapped Euclidean supermembrane configurations over a three-torus. Nonzero-flux sectors show the contribution from Kaluza-Klein states of supergravitons dissolved in the wrapped supermembrane, which is also consistent to the U-duality. It should be remarked that in eq. (4.3) the three-dimensional $\mathcal{N}_{3}=2$ theory can not be described as a cohomological field theory by the twisting procedure because the theory has only a single Higgs field. The twisting needs at least two Higgs fields. So it is possible that quantum fluctuations contribute nontrivially to the partition function of the $\mathcal{N}_{3}=2$ theory. In other words, the Higgs field yields a continuous spectrum, and thus it is likely that the cancelation of the contributions from the Higgs and its superpartner is not precisely realized, which is analogous to the situation of the appearance of the deficit terms in the Witten index calculation for super Yang-Mills quantum mechanics [9, 10]. As mentioned at the beginning of this section, this situation forms a sharp contrast with the matrix string case. It seems to be a reason of the failure of the quasi-classical approach and at the same time to show a crucial difference between strings and membranes. 
It would be interesting to speculate how the result changes when the continuous zero-modes of the Higgs and its superpartner are removed. Let us consider the $\mathcal{N}_{3}=2$ supersymmetric SU(n) gauge theory with the twisted boundary conditions [17]:

$$
\begin{aligned}
A_{a}\left(\sigma_{1}, \sigma_{2}, \sigma_{3}\right) & =A_{a}\left(\sigma_{1}+R_{1}, \sigma_{2}, \sigma_{3}\right) \\
& =P A_{a}\left(\sigma_{1}, \sigma_{2}+R_{2}, \sigma_{3}\right) P^{-1} \\
& =Q A_{a}\left(\sigma_{1}, \sigma_{2}, \sigma_{3}+R_{3}\right) Q^{-1},
\end{aligned}
$$

where $P$ and $Q$ are 't Hooft clock and shift matrices satisfying $P Q P^{-1} Q^{-1} \in \mathbb{Z}_{n}$. All the other fields also obey the same boundary conditions. In this case, the zero-modes of the Higgs field are absent, and the spectrum is discrete. The partition function is equal to the Witten index, which is known to be 1 from ref. [25]. Thus the value of the partition function of the non-Abelian part without the zero-modes of the Higgs multiplet will become

$$
Z_{\mathcal{N}_{3}=8}^{\mathrm{SU}(\mathrm{N}) / \mathbb{Z}_{\mathrm{N}}}=\left.\frac{1}{N^{2}} \sum_{N=m n} m^{2} Z_{\mathcal{N}_{3}=2}^{\mathrm{SU}(\mathrm{n})}\right|_{\text {twisted b.c. }}=\frac{1}{N^{2}} \sum_{m \mid N} m^{2} .
$$

This reproduces the measure factor entering D-instanton (supergraviton in the context of M-theory) effects [9, 10, 11, 20].

\section{Discussion}

The analysis of the dynamics of wrapped supermembranes has already been the subject of various papers [26, 27], but a complete understanding of the supermembrane as a fundamental object is still laking (despite an interesting recent attempt [1]). The main difficulties rely on needle-like deformations that cost no energy [28], which are likely to survive after compactifications [27]. As an additional complication, a naive generalization of the matrix model regularization [29] of the flat space light-cone supermembrane was shown to fail [27], because of the difficulty for the structure constants to satisfy both the Jacobi identity and the periodicity conditions around the compact directions. Consequently, strictly speaking, a direct and rigorous derivation of the matrix membrane model (2.1) from wrapped supermembranes on a three-torus is not known.

We showed in this paper that the membrane matrix model contains enough information for reproducing the measure factor of wrapped supermembranes, and exhibiting the full $S l(3, \mathbb{Z}) \times S l(2, \mathbb{Z})$ symmetries of the moduli space of the model. Again a direct analysis of these symmetries from the perspective of the supermembrane world-volume action turns to be more subtle than the naive generalization of string approach [30,1]. 
It is remarkable that turning on/off the continuous zero-modes of the Higgs multiplet in the $\mathcal{N}_{3}=2$ theory lead to respectively the supermembrane instantons measure factor and D-instanton (supergraviton) measure factor. The former corresponds to the system of the bound states of the supermembrane and supergravitons, and the latter to the system of the supergraviton states alone. This is again suggestive of the Higgs zero-modes being identified with an essential ingredient of the supermembrane.

Differently from the matrix string case [19], where by dimensional analysis one can be convinced of the appearance of a conformal field theory description in the infra-red limit, we will not be able to expect completely analogous phenomena for membranes. This is supported by the failure of the infra-red (strong coupling) limit analysis of section 3 to reproduce the correct measure factor (1.4). Consequently, reconstructing the interactions between supermembranes will take a different route than in [31.6 Rederiving these results from a direct supermembrane path integral analysis is an important problem, that is left for a future publication.

Acknowledgements: We thank Adi Armoni, Shyamoli Chaudhuri, Francois David, Hiroyuki Fuji, Masafumi Fukuma, Nobuyuki Ishibashi, Elias Kiritsis, Ivan Kostov, Tsunehide Kuroki, Tadakatsu Sakai and Tamiaki Yoneya for very useful discussions, as well as Jan Plefka for email correspondence. P.V. thanks all the participants of the superstring theory meeting and the people of the physics department of the university of Crete at Heraklion for discussions. Also, F.S. thanks participants and organizers of the string theory symposium at Tohwa university, Fukuoka, Japan, and members of theory group at KEK for discussions and hospitality during his stay. P.V. received partial support from the EEC contract HPRN-CT-2000-00122.

6 For a recent interesting attempt toward perturbative formulation for membranes, see ref. [32]. 


\section{References}

[1] B. Pioline, H. Nicolai, J. Plefka and A. Waldron, $R^{4}$ couplings, the fundamental membrane and exceptional theta correspondences, JHEP 03 (2001) 036, hep-th/0102123.

[2] E. Cremmer, B. Julia and J. Scherk, Supergravity Theory in Eleven-Dimensions, Phys. Lett. B76 (1978) 409.

[3] E. Bergshoeff, E. Sezgin and P.K. Townsend, Supermembranes and Eleven-Dimensional Supergravity, Phys. Lett. 189B (1987) 75.

[4] P.K. Townsend, Four Lectures on M-theory, hep-th/9612121.

[5] P.K. Townsend, The Eleven-Dimensional Supermembrane Revisited, Phys. Lett. B350 (1995) 184, hep-th/9501068;

E. Witten, String Theory Dynamics in Various Dimensions, Nucl. Phys. B443 (1995) 85, hep-th/9503124.

[6] M.B. Green and M. Gutperle, Effects of D-instantons, Nucl. Phys. B498 (1997) 195, hep-th/9701093.

[7] E. Kiritsis and B. Pioline, On $R^{4}$ threshold corrections in IIB string theory and $(p, q)$ string instantons, Nucl. Phys. B508 (1997) 509, hep-th/9707018;

C. Bachas, C. Fabre, E. Kiritsis, N.A. Obers and P. Vanhove, Heterotic / type I duality and D-brane instantons, Nucl. Phys. B509 (1998) 33, hep-th/9707126.

[8] B. Pioline and N.A. Obers, U-duality and M-theory, Phys. Rept. 318 (1999) 113, hep-th/9809039.

[9] P. Yi, Witten index and threshold bound states of D-branes, Nucl. Phys. B505 (1997) 307, hep-th/9704098.

[10] S. Sethi and M. Stern, D-brane Bound States Redux, Commun. Math. Phys. 194 (1998) 675, hep-th/9705046.

[11] M.B. Green and M. Gutperle, D-particle bound states and the D-instanton measure, JHEP 9801 (1998) 005, hep-th/9711107.

[12] N. Dorey, T. J. Hollowood and V. V. Khoze, Notes on soliton bound-state problems in gauge theory and string theory, hep-th/0105090.

[13] W. Taylor, D-brane field theory on compact spaces, Phys. Lett. B394 (1997) 282, hep-th/9611042.

[14] B. de Wit, J. Hoppe and H. Nicolai, On the quantum mechanics of supermembranes, Nucl. Phys. B305 (1988) 545.

[15] O.J. Ganor, S. Ramgoolam and W. Taylor, Branes, fluxes and duality in M(atrix)theory, Nucl. Phys. B492 (1997) 191, hep-th/9611202.

[16] I.K. Kostov and P. Vanhove, Matrix string partition functions, Phys. Lett. B444 (1998) 196, hep-th/9809130.

[17] F. Sugino, Cohomological field theory approach to matrix strings, Int. J. Mod. Phys. A14 (1999) 3979, hep-th/9904122. 
[18] N.A. Obers and B. Pioline, Eisenstein series in string theory, Class. Quant. Grav. 17 (2000) 1215, hep-th/9910115.

[19] R. Dijkgraaf, E. Verlinde and H. Verlinde, Matrix String Theory, Nucl. Phys. B500 (1997) 43, hep-th/9703030.

[20] G. Moore, N. Nekrasov and S. Shatashvili, D-particle bound states and generalized instantons, Commun. Math. Phys. 209 (2000) 77, hep-th/9803265.

[21] P. Austing, The cohomological supercharge, JHEP 01 (2001) 009,hep-th/0011211.

[22] M. Porrati and A. Rozenberg, Bound States at Threshold in Supersymmetrical Quantum Mechanics, Nucl. Phys. B515 (1998) 184, hep-th/9708119.

[23] C. Vafa and E. Witten, A Strong coupling test of $S$ duality, Nucl. Phys. B431 (1994) 3, hep-th/9408074.

[24] E. Witten, Constraints on Supersymmetry Breaking, Nucl. Phys. B202 (1982) 253.

[25] I. Affleck, J.A. Harvey and E. Witten, Instantons And (Super)Symmetry Breaking In (2+1)-Dimensions, Nucl. Phys. B206 (1982) 413.

[26] J. G. Russo, Supermembrane dynamics from multiple interacting strings, Nucl. Phys. B492 (1997) 205, hep-th/9610018.

[27] B. de Wit, K. Peeters and J.C. Plefka, The Supermembrane with winding, Nucl. Phys. Proc. Suppl. 62 (1998) 405, hep-th/9707261;

B. de Wit, K. Peeters and J. Plefka, Supermembranes with winding, Phys. Lett. B409 (1997) 117, hep-th/9705225.

[28] B. de Wit, M. Luscher and H. Nicolai, The Supermembrane is Unstable, Nucl. Phys. B320 (1989) 135.

[29] B. de Wit, U. Marquard and H. Nicolai, Area Preserving Diffeomorphisms And Supermembrane Lorentz Invariance, Commun. Math. Phys. 128 (1990) 39.

[30] A. Lukas and B. A. Ovrut, U-duality symmetries from the membrane world-volume, Nucl. Phys. B502 (1997) 191, hep-th/9704178.

[31] T. Wynter, Gauge Fields and Interactions in Matrix String Theory, Phys. Lett. B415 (1997) 349, hep-th/9709029.

[32] M. Hayakawa and N. Ishibashi, Perturbative world-volume dynamics of the bosonic membrane and string, hep-th/0107103. 